\title{
AdaMKL: A Novel Biconvex Multiple Kernel Learning Approach
}

\author{
Ziming Zhang, Ze-Nian Li, Mark Drew \\ School of Computing Science, Simon Fraser University, Vancouver, B.C., Canada \\ \{zza27, li,mark\}@cs.sfu.ca
}

\begin{abstract}
In this paper, we propose a novel large-margin based approach for multiple kernel learning (MKL) using biconvex optimization, called Adaptive Multiple Kernel Learning (AdaMKL). To learn the weights for support vectors and the kernel coefficients, AdaMKL minimizes the objective function alternatively by learning one component while fixing the other at a time, and in this way only one convex formulation needs to be solved. We also propose a family of biconvex objective functions with an arbitrary $\ell_{p}$-norm $(p \geq 1)$ of kernel coefficients. As our experiments show, AdaMKL performs comparably with the state-of-the-art convex optimization based MKL approaches, but its learning is much simpler and faster.
\end{abstract}

\section{Introduction}

Multiple Kernel Learning (MKL) [1] aims to generate an optimal kernel automatically by combining a set of basic kernels linearly as well as learn the weights for support vectors simultaneously. Given $N$ labeled data $\mathcal{D}=\left\{\left(\mathbf{x}_{i}, y_{i}\right)\right\}_{i=1, \cdots, N}$ where $\forall i, \mathbf{x}_{i} \in \mathcal{X}$ is an input feature vector and $y_{i} \in\{+1,-1\}$ is its class label for binary classification, and $M$ feature mapping functions $\Phi=\left\{\phi_{j}\right\}_{j=1, \cdots, M}$, each of which maps an input feature into a Hilbert space $\mathcal{H}_{j}$, a reproducing kernel $K_{j}$ is defined as an inner product of $\phi_{j}\left(\mathbf{x}_{m}\right)$ and $\phi_{j}\left(\mathbf{x}_{n}\right)$, that is, $K_{j}\left(\mathbf{x}_{m}, \mathbf{x}_{n}\right)=\left\langle\phi_{j}\left(\mathbf{x}_{m}\right), \phi_{j}\left(\mathbf{x}_{n}\right)\right\rangle_{\mathcal{H}_{j}}$, and the optimal kernel $K_{\text {opt }}$ learned by MKL can be represented as Eqn. 1, where $\gamma$ are kernel coefficients.

$$
K_{\text {opt }}=\sum_{j=1}^{M} \gamma_{j} K_{j}, \quad \forall j, \gamma_{j} \geq 0
$$

By putting MKL into the context of large-margin classifiers, e.g. support vector machines (SVM), several MKL approaches have been proposed [1, 4, 5, 7, 2, $3,6]$. Typically the objective functions in the existing approaches are convex, with the norm constraint of $\gamma$, e.g. $\|\gamma\|_{1}=1$ (sparse learning) $[1,4,5,7,6]$, or $\|\gamma\|_{2}=1$ (non-sparse learning) [2], or even $\|\gamma\|_{p}=1(p \geq 1)$ [3]. Also, different optimization approaches have been used in MKL, e.g. second-order cone programming [1], semi-definite programming [4], semi-infinite programming $[7,2]$, gradient-based approaches $[5,3,6]$.

Commonly, learning kernel coefficients efficiently is one of the major difficulties in MKL due to the existence of the norm constraint and non-negative constraint of kernel coefficients. In this paper, we propose a biconvex optimization based MKL approach, named Adaptive Multiple Kernel Learning (AdaMKL). A function $f: X \times Y \rightarrow \mathbb{R}$ is called biconvex if $f$ is convex both in $x \in X$ for fixed $y \in Y$ and in $y \in Y$ for fixed $x \in X$. The main contribution of this paper is that we propose a family of biconvex, rather than convex, objective functions to learn the parameters in MKL by introducing an arbitrary $\ell_{p}$-norm $(p \geq 1)$ constraint into the objective function so that the norm constraint and non-negative constraint are hidden in the dual of AdaMKL when learning the weights of support vectors without consideration explicitly. It turns out that the traditional sparse (or non-sparse) learning due to the norm constraint is separated, which makes the learning of AdaMKL much simpler and faster compared to the convex optimization based MKL approaches.

The rest of this paper is organized as follows. Section 2 describes our approach in detail. Section 3 shows our experimental results. We finally conclude the paper in Section 4.

\section{Adaptive Multiple Kernel Learning}

In this paper, we focus on the binary classification using AdaMKL. We follow the notations in Section 1.

\subsection{Motivation}

Eqn. 2 shows one type of common decision functions in MKL, where $\theta$ are the coefficients w.r.t. kernels, w 
are the normal vectors for SVM, and $b$ is the bias term.

$$
f(\mathbf{x})=\sum_{m=1}^{M} \theta_{m}\left\langle\mathbf{w}_{m}, \phi_{m}(\mathbf{x})\right\rangle+b
$$

If letting $\theta_{m} \geq 0, \mathbf{w}_{m}^{\prime}=\sqrt{\theta_{m}} \mathbf{w}_{m}$ and fitting Eqn. 2 into the original SVM formulation, Kloft et al. [3] showed that the corresponding MKL formulation is actually a convex optimization problem (OP). However, if directly fitting Eqn. 2 into an SVM without any constraint on $\theta$, we can obtain Eqn. 3, where $C$ is a constant and $\xi$ are errors.

$$
\begin{aligned}
\min _{\theta, \mathbf{w}, b, \xi} & \frac{1}{2} \sum_{m} \theta_{m}^{2}\left\|\mathbf{w}_{m}\right\|_{2}^{2}+C \sum_{i} \xi_{i} \\
\text { s.t. } & \forall i, y_{i}\left[\sum_{m} \theta_{m}\left\langle\mathbf{w}_{m}, \phi_{m}\left(\mathbf{x}_{i}\right)\right\rangle+b\right] \geq 1-\xi_{i} \\
& \xi_{i} \geq 0, C \geq 0
\end{aligned}
$$

Clearly, Eqn. 3 defines a biconvex OP, and its objective function can be easily relaxed to one of its upper bounds as shown in Eqn. 4, which also defines a biconvex OP and allows the introduction of an arbitrary $\ell_{p}$-norm of kernel coefficients into AdaMKL.

$$
\begin{aligned}
\sum_{m} \theta_{m}^{2}\left\|\mathbf{w}_{m}\right\|_{2}^{2} & \leq\left\|\theta^{2}\right\|_{p}\|\mathbf{w}\|_{2}^{2} \\
& \leq\left\|\theta^{2}\right\|_{1}\|\mathbf{w}\|_{2}^{2} \leq\|\theta\|_{1}^{2}\|\mathbf{w}\|_{2}^{2}
\end{aligned}
$$

\subsection{Formulation and Optimization}

We define the primal of our AdaMKL as Eqn. 5, which contains a family of biconvex objective functions, and learn $\mathbf{w}$ and $\theta$ alternatively.

$$
\begin{aligned}
\min _{\theta, \mathbf{w}, b, \xi} & \frac{1}{2} \mathcal{N}_{p}(\theta)\|\mathbf{w}\|_{2}^{2}+C \sum_{i} \xi_{i} \\
\text { s.t. } & \forall i, y_{i}\left[\sum_{m} \theta_{m}\left\langle\mathbf{w}_{m}, \phi_{m}\left(\mathbf{x}_{i}\right)\right\rangle+b\right] \geq 1-\xi_{i} \\
& \xi_{i} \geq 0, C \geq 0 \\
\text { where } & \mathcal{N}_{0}(\theta)=\|\theta\|_{1}^{2}, \mathcal{N}_{p}(\theta)=\left\|\theta^{2}\right\|_{p \geq 1}
\end{aligned}
$$

\subsubsection{Learning Parameter $w$}

When learning parameter $\mathbf{w}$, parameter $\theta$ is fixed. Using Lagrange multipliers, we rewrite our primal in Eqn. 5 and obtain its dual in Eqn. 6, where $\alpha$ is the set of Lagrange multipliers. Accordingly, we can calculate each $\mathbf{w}_{m}$ using Eqn. 7 and rewrite Eqn. 1 as Eqn. 8.

$$
\begin{array}{cl}
\max _{\alpha} & \sum_{i} \alpha_{i}-\frac{1}{2} \sum_{i, j, m} \alpha_{i} \alpha_{j} y_{i} y_{j} \frac{\theta_{m}^{2}}{\mathcal{N}_{p}(\theta)} K_{m}\left(\mathbf{x}_{i}, \mathbf{x}_{j}\right) \\
\text { s.t. } & \forall i, 0 \leq \alpha_{i} \leq C, \quad \sum_{i} \alpha_{i} y_{i}=0
\end{array}
$$

$$
\begin{gathered}
\mathbf{w}_{m}=\sum_{i} \alpha_{i} y_{i} \frac{\theta_{m}}{\mathcal{N}_{p}(\theta)} \phi_{m}\left(\mathbf{x}_{i}\right) \\
K_{\text {opt }}=\sum_{m} \gamma_{m} K_{m}=\sum_{m} \frac{\theta_{m}^{2}}{\mathcal{N}_{p}(\theta)} K_{m}
\end{gathered}
$$

Notice that if $\mathcal{N}_{p}(\theta)=\left\|\theta^{2} \quad\right\|_{p \geq 1}$ and we denote $\gamma_{m}=\frac{\theta_{m}^{2}}{\mathcal{N}_{p}(\theta)}$, then we actually obtain $\|\gamma\|_{p}=1$. Therefore, an arbitrary $\ell_{p}$-norm constraint of kernel coefficients is hidden in the dual formulation without consideration explicitly, and since $\forall m, \frac{\theta_{m}^{2}}{\mathcal{N}_{p}(\theta)} \geq 0$ always holds, it is guaranteed that $K_{\text {opt }}$ is a valid kernel as long as $\forall m, K_{m}$ is valid.

\subsubsection{Learning Parameter $\theta$}

We employ linear programming (LP) or quadratic programming $(\mathrm{QP})$ to learn parameter $\theta$ while fixing parameter $w$, and the corresponding primal formulations are shown in Eqn. 9.

$$
\begin{aligned}
\min _{\theta, b, \xi} & \mathcal{L}_{n}(\theta)+C \sum_{i} \xi_{i} \\
\text { s.t. } & \forall i, y_{i}\left[\sum_{m} \theta_{m} \phi_{m}^{\mathbf{w}}\left(\mathbf{x}_{i}\right)+b\right] \geq 1-\xi_{i} \\
& \xi_{i} \geq 0, C \geq 0, n=1,2 \\
\text { where } & \mathcal{L}_{1}(\theta)=\sum_{m}\left|\theta_{m}\right|, \mathcal{L}_{2}(\theta)=\frac{1}{2} \sum_{m} \theta_{m}^{2} \\
& \phi_{m}^{\mathbf{w}}\left(\mathbf{x}_{i}\right)=\left\langle\mathbf{w}_{m}, \phi_{m}\left(\mathbf{x}_{i}\right)\right\rangle
\end{aligned}
$$

To summarize our optimization algorithm, Algorithm 1 lists all the details as follows.

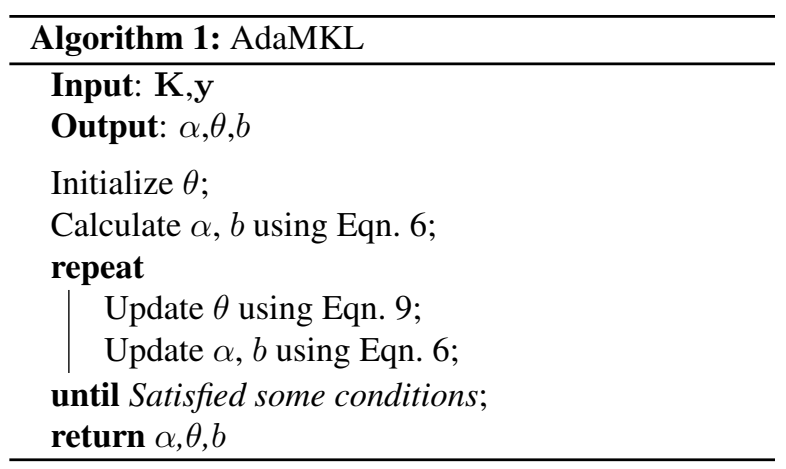

\subsection{Discussion}

Our AdaMKL shares the same computational complexity as LP or QP, and in our implementation we set the initial value of $\theta$ as $\mathbf{1}$ as did in some other convex optimization based MKL approaches (e.g. [6], [5]) for comparison. 
Another important issue for AdaMKL is its convergency, because this could be an important termination criterion. Generally speaking, we cannot guarantee that any formulation of AdaMKL will converge to its local minimum finally. However, letting $g_{1}\left(\theta, \mathbf{w}, \xi_{1}\right)=$ $\min \left\{\frac{1}{2} \mathcal{N}_{p}(\theta)\|\mathbf{w}\|_{2}^{2}+C \sum_{i} \xi_{1, i}\right\}$ and $g_{2}\left(\theta, \xi_{2}\right)=$ $\min \left\{\mathcal{L}_{n}(\theta)+C \sum_{i} \xi_{2, i}\right\}$, in the following cases it is guaranteed that AdaMKL will converge.

Proposition 1. For hard-margin cases $(C=+\infty)$, if Eqn. 6 can be solved at the initialization stage, then AdaMKL will converge to a local minimum.

Proof. In this case, after each update, we have $\xi_{1}=\mathbf{0}$. Then considering the primal, at the $t^{t h}$ and $s^{t h}$ updates for $\theta$ and $\mathbf{w}$, respectively, we have $g_{1}\left(\theta^{(t)}, \mathbf{w}^{(s)}, \xi_{1}^{(s)}\right) \geq$ $g_{1}\left(\theta^{(t)}, \mathbf{w}^{(s+1)}, \xi_{1}^{(s+1)}\right) \geq g_{1}\left(\theta^{(t+1)}, \mathbf{w}^{(s+1)}, \xi_{1}^{(s+1)}\right)$ plus $\forall\left(\theta, \mathbf{w}, \xi_{1}\right), g_{1} \geq 0$, so AdaMKL will converge to a local minimum.

Proposition 2. If $g_{2}$ converged, then AdaMKL has converged to a local minimum.

Proof. Suppose at the $t^{t h}$ and $s^{t h}$ updates for $\theta$ and $\mathbf{w}$, respectively, $g_{2}$ converged, then $\theta^{(t)}=\theta^{(t+1)}$ and $\xi_{1}^{(s)}=\xi_{2}^{(t)}=\xi_{2}^{(t+1)}=\xi_{1}^{(s+1)}$. Due to the local convergency, we have $g_{1}\left(\theta^{(t)}, \mathbf{w}^{(s)}, \xi_{1}^{(s)}\right)=$ $g_{1}\left(\theta^{(t+1)}, \mathbf{w}^{(s)}, \xi_{2}^{(t+1)}\right)=g_{1}\left(\theta^{(t+1)}, \mathbf{w}^{(s+1)}, \xi_{1}^{(s+1)}\right)$, which indicates AdaMKL has converged.

\section{Experiments}

We construct four different specific formulations of AdaMKL, that is, AdaMKL- $\mathcal{N}_{0} \mathcal{L}_{1}$, AdaMKL- $\mathcal{N}_{1} \mathcal{L}_{1}$, AdaMKL- $\mathcal{N}_{1} \mathcal{L}_{2}$, AdaMKL- $\mathcal{N}_{2} \mathcal{L}_{2}$, where $\mathcal{N}$ and $\mathcal{L}$ follow the notations in Eqn. 5 and 9, respectively.

\subsection{Toy Example}

We first tested our approach on a toy dataset, where data is sampled randomly based on 2D Gaussian distributions. For positive data, the mean vector of the Gaussian distribution is $[00]$ and the covariance matrix is $[0.30 ; 00.3]$. For negative data, the mean vectors of the Gaussian distributions are [-1 -1] and [1 1], and the covariance matrices are $[0.10 ; 00.1]$ and $[0.20 ; 00.2]$, respectively. Each distribution generates 100 samples. We create 10 Gaussian kernels with parameters from 1 to 10 , step by 1 . Here, we fix the parameter $\mathrm{C}$ in AdaMKL as $10^{5}$ without tuning.

Fig. 1 shows the behavior of the dual values of Eqn. 6 at each update using different AdaMKL. Since there are always some misclassified instances in this toy example, Prop. 1 cannot be demonstrated here and therefore

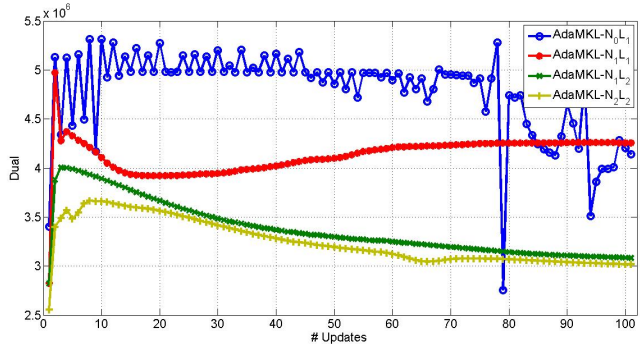

Figure 1. Behaviors of dual values of Eqn. 6 for the four different AdaMKL. This figure is best viewed in color.
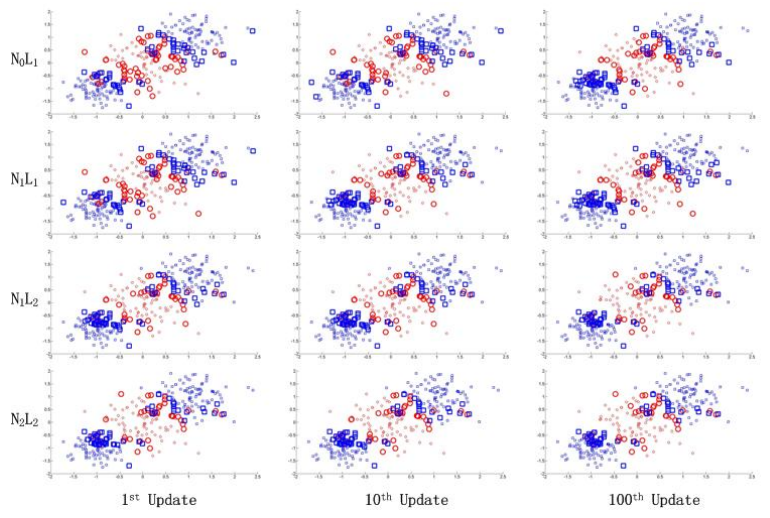

Figure 2. Illustration of the distributions of support vectors after 1, 10, 100 updates for different AdaMKL. Red circles denote positive data, and blue squares denote negative data. Bigger circles and squares denote the support vectors. This figure is best viewed in color.

their convergency behaviors are difficult to predict, especially for AdaMKL- $\mathcal{N}_{0} \mathcal{L}_{1}$. However, from this figure we can also see that: (1) The norm constraint makes AdaMKL converge to a local minimum more smoothly. (2) In general, most of the biggest changes occur in the first few updates, which indicates that our AdaMKL can be terminated in a few updates. In Fig. 2 we can see that for each of the four AdaMKL, the support vector distribution changes slightly after the $1^{\text {st }}$ update compared to the others. This also suggests that an early stop strategy can be applied in our AdaMKL to speed up its learning.

\subsection{Benchmark Datasets}

We also tested our AdaMKL on four benchmark datasets ${ }^{1}$ : breast-cancer, heart, thyroid, and titanic. Each dataset contains 100 pairs of training and test files, where the numbers of training patterns are 200, 170, 140,150 , the numbers of test patterns are 77, 100, 75, 2051 , and the feature dimensions are $9,13,5,3$, respec-

\footnotetext{
${ }^{1}$ These datasets can be downloaded from http://ida. first.fraunhofer.de/projects/bench/.
} 


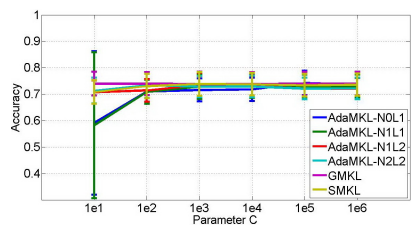

(a) Breast-Cancer

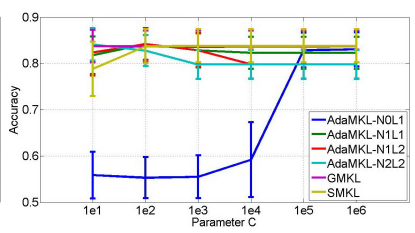

(b) Heart

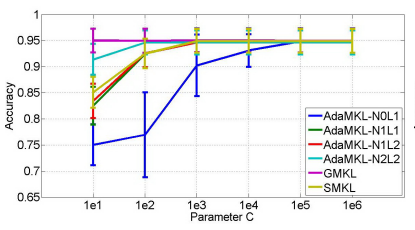

(c) Thyroid

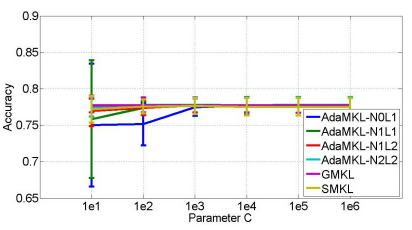

(d) Titanic

Figure 3. Accuracy comparison between the six MKL approaches on the four benchmark datasets. This figure is best viewed in color.

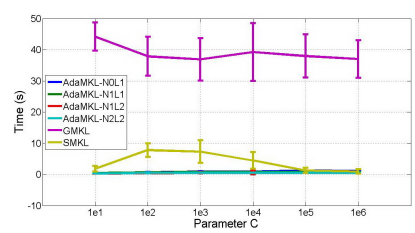

(a) Breast-Cancer

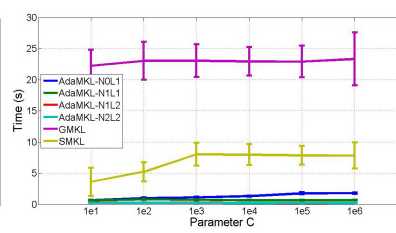

(b) Heart

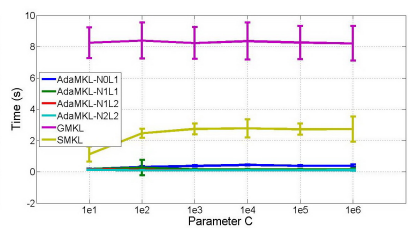

(c) Thyroid

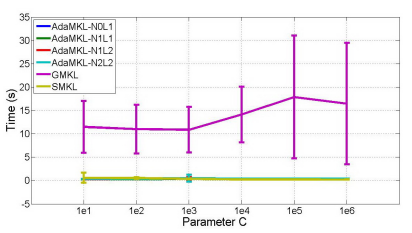

(d) Titanic

Figure 4. Time comparison between the six MKL approaches on the four benchmark datasets. This figure is best viewed in color.

tively. The kernels used in our experiments are Gaussian kernels with parameters from 0.02 to 0.14 step by 0.02 , and polynomial kernels with parameters $1,2,3$, respectively. We constructed kernels using both features and their element at each dimension, resulting in 100, 140, 60, 40 kernels for the corresponding datasets.

Fig. 3 and 4 show the comparison in terms of accuracy and computational time, respectively, using different parameter $C$ between AdaMKL and the other two state-of-the-art convex optimization based MKL approaches: SimpleMKL ${ }^{2}$ (SMKL) [6], and General MKL $^{3}$ (GMKL) [5]. All the implementations of different approaches are based on Matlab with the same quadratic programming solver, and run on a $2.33 \mathrm{GHz}$ Core 2 Duo CPU. As we see, in general, for all the datasets, each AdaMKL performs comparably with the other MKL approaches (so does AdaMKL- $N_{0} L_{1}$ when $C$ is bigger), but usually takes much less computational time, and they are all comparable to the methods listed in the dataset web site (Breast-Cancer: [69.64 75.23], Heart: [79.71 84.05], Thyroid: [95.20 95.80], Titanic: [76.02 77.58]).

\section{Conclusion}

In this paper, Adaptive Multiple Kernel Learning (AdaMKL) is proposed based on the large-margin criterion. We propose a family of biconvex objective functions for AdaMKL which are minimized by updating

\footnotetext{
${ }^{2}$ Its code is downloaded from http://asi.insa-rouen. fr/enseignants/ arakotom/code/mklindex.html.

${ }^{3}$ Its code is downloaded from http://research. microsoft.com/en-us/um/people/manik/code/GMKL/ download.html.
}

the parameters alternatively. As a result, an arbitrary $\ell_{p^{-}}$ norm $(p \geq 1)$ of kernel coefficients can be involved in our formulation, and the sparse (or non-sparse) learning is separated from this norm constraint, which makes the learning process much easier and faster. We mainly discuss the convergency issue of AdaMKL, and compare four specific AdaMKL with two state-of-the-art convex optimization based MKL approaches in our experiments. In general, the performance of each AdaMKL is comparable to the MKL approaches with much faster learning speed.

\section{References}

[1] F. R. Bach, G. R. G. Lanckriet, and M. I. Jordan. Multiple kernel learning, conic duality, and the smo algorithm. In ICML'04, page 6, New York, NY, USA, 2004. ACM.

[2] M. Kloft, U. Brefeld, P. Laskov, and S. Sonnenburg. Nonsparse multiple kernel learning. In NIPS Workshop on Kernel Learning: Automatic Selection of Optimal Kernels, 2008.

[3] M. Kloft, U. Brefeld, S. Sonnenburg, P. Laskow, K.-R. Mueller, and A. Zien. Efficient and accurate $\ell_{p}$-norm multiple kernel learning. In NIPS'09, 2009.

[4] G. Lanckriet, N. Cristianini, P. Bartlett, and L. E. Ghaoui. Learning the kernel matrix with semidefinite programming. JMLR, 5:27-72, 2004.

[5] A. Rakotomamonjy, F. Bach, S. Canu, and Y. Grandvalet. More efficiency in multiple kernel learning. In ICML'07, pages 775-782, 2007.

[6] A. Rakotomamonjy, F. Bach, S. Canu, and Y. Grandvalet. SimpleMKL. JMLR, 9:2491-2521, 2008.

[7] S. Sonnenburg, B. S. Bernhard, P. Bennett, and E. Parrado-Hernández. Large scale multiple kernel learning. JMLR, 7, 2006. 